\title{
Fertilization by Intracytoplasmic Sperm Injection and Subsequent Embryo Development In Vitro to Blastocysts in Japanese Monkey (Macaca fuscata)
}

\author{
Yoshihiko Hosoi ${ }^{1 *}$, Ryuzo Torii ${ }^{2}$, Nahoko Fujinami ${ }^{1}$, \\ Kazuya Matsumoto ${ }^{1}$, Kazuhiro Saeki ${ }^{1}$ and Akira Iritani ${ }^{1}$ \\ ${ }^{1}$ Department of Genetic Engineering, Faculty of Biology Oriented Science \& Technology, Kinki \\ University, 930 Nishimitani Uchita, Wakayama 649-6493, Japan \\ ${ }^{2}$ Research Institute of Experimental Animal, Shiga University of Medical Science, Otsu, Shiga 520- \\ 2192, Japan
}

\begin{abstract}
Intracytoplasmic Sperm Injection (ICSI) has been widely applied for curing human infertility. In this study the developmental potential of Japanese monkey embryos produced by ICSI is reported in a practically relevant system. Oocytes retrieved by laparoscopy from follicles in ovaries of gonadotrophin-stimulated fertile females were fertilized by ICSI using spermatozoa obtained from a fertile male. An additional chemical stimulus was not necessary to achieve oocyte activation with pronuclear formation after ICSI. Successful fertilization was confirmed by extrusion of the second polar body and the presence of both male and female pronuclei at 18-20 h post-ICSI. Some two-cell stage embryos obtained by ICSI were transferred to synchronous recipients and the others were cultured in CMRL medium for $168 \mathrm{~h}$ to assess their developmental competence. Oocytes collected laparoscopically from hyper-stimulated monkey ovaries were fertilized by ICSI and completed preimplantation development in vitro, however no pregnancy was confirmed after embryo transfer. This study demonstrates for the first time that the oocytes of the Japanese monkey are able to support advanced embryonic preimplantation development in vitro. It is suggested that the Japanese monkey is an excellent preclinical model for examining and understanding many aspects of ICSI for endangered primates.
\end{abstract}

Key words: Assisted reproduction, Fertilization, ICSI,

Received: March 28, 2002

Accepted: February 18, 2003

*To whom correspondence should be addressed.

e-mail: hosoi@gene.waka.kindai.ac.jp

\section{Laparoscopy, Macaca fuscata}

The introduction of intracytoplasmic sperm injection (ICSI) has revolutionized methods of human assisted reproduction [1]. Originally this technique was developed for a study to elucidate the mechanism of fertilization in mammals. A rabbit was successfully used to produce the first offspring by ICSI [2], but rabbits have not been routinely used as a model for studying ICSI. Subsequently ICSI has been used to generate live offspring of humans [1], rhesus monkeys [3], sheep [4], swine [5], bovine [6], equine [7], and murine [8]. Non-human primates represent a valuable resource for studying human reproduction. The techniques of gamete manipulation, in vitro fertilization embryonic development, embryo transfer and implantation have been well documented for a variety of non-human primates [9-15], which are considered good models for studying human reproduction. The first report of ICSI in a non-human primate demonstrated limited success using in-vitro matured rhesus oocytes isolated from females which has underwent ovariectomy [14]. The Japanese monkey is a domestic non-human primate and become a laboratory animal candidate, recently. However, the birth of a live Japanese monkey as a result of ICSI has not yet been reported.

The objectives of this study were to investigate the developmental ability of mature Japanese monkey oocytes, collected by laparoscopy from gonadotrophinstimulated females, fertilized by ICSI and to assess their embryonic developmental competence. 


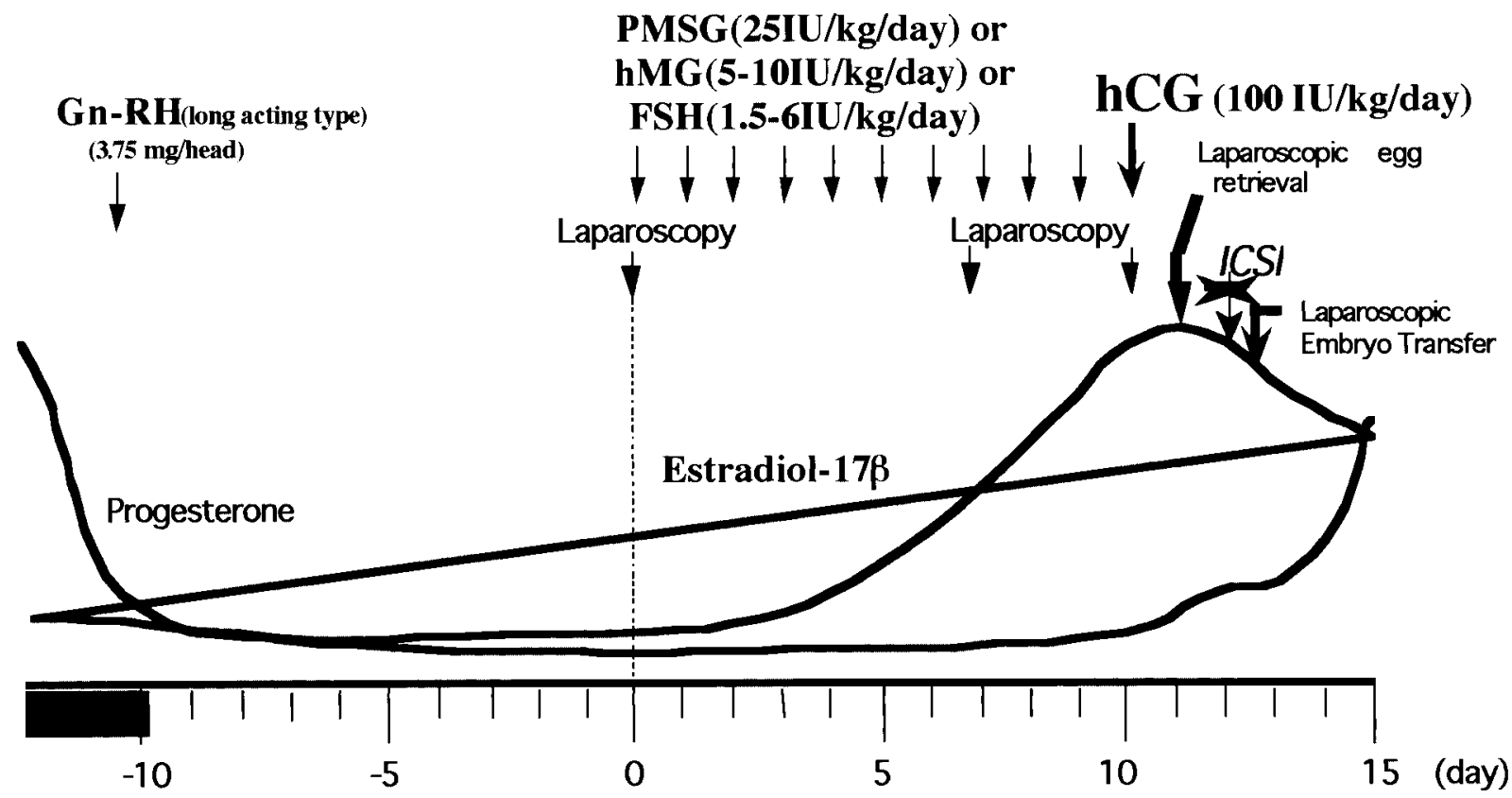

Fig. 1. Time course of superovulation, egg retrieval and ICSI-ET in the Japanese monkey. This figure showed an administration of hormones for superovulation, and a precise timing of ICSI and ET according to the estrus cycle of Japanese monkey in mating season. It was explained in materials and methods.

\section{Materials and Methods}

Oocyte and sperm collection

Hyperstimulation of female Japanese macaques (Macaca fuscata) exhibiting normal menstrual cycles was induced by a regimen of exogenous gonadotrophic hormones [15]. In this study, the protocol of ovarian stimulation, egg collection, fertilization by ICSI and embryo transfer was done in the mating season (October to March) and the schedule of the treatment is shown in Fig. 1. In brief, females were treated by injections of a long acting gonadotrophin-releasing hormone $(\mathrm{GnRH})$ agonist, (Leuprin; Takeda Pharm. Co., Ltd., Japan) $3.75 \mathrm{mg} /$ body weight and then pregnant mare serum (PMSG: Serotoropin, Teikokuzoki Co., Ltd., Japan) was administered once daily (25 IU, i.m.) for 9 days for follicle recruitment. Laparoscopic observation was performed on day 7 to confirm an existence of adequate follicles in an ovary under anesthesia with xylasin hydrochloride $(1 \mathrm{mg} / \mathrm{kg}$ : Celactal, Byer Co., Ltd., Japan) and ketamin hydrochloride ( $5 \mathrm{mg} / \mathrm{kg}$ : Ketaral, Sankyo Co., Ltd., Japan). Tenth day after first PMSG injection, injection i.m. of $100 \mathrm{lU} / \mathrm{kg} / \mathrm{body}$ hCG (Puberogen, Sankyozoki Co., Ltd., Japan) was performed. In case of repeated hyperstimulation, hMG (Pergonal, Teikokuzouki, Co.,
Ltd., Japan) or FSH (Antorin: Denkaseiyaku, Co., Ltd., Japan) was used for follicle recruitment instead of PMSG. Follicles were aspirated laparoscopically at 40 $\mathrm{h}$ post-hCG injection and the collected oocytes were immediately assessed for nuclear maturity. This procedure was conducted by the method reported previously [15]. Immature oocytes that had undergone germinal vesicle breakdown (GVBD), not matured to the second metaphase of meiotic division, were cultured at $39^{\circ} \mathrm{C}$ in a pre-equilibrated micro-drop of modified TALP medium. Mature oocytes were cultured for up to $2-3 \mathrm{~h}$ in pre-equilibrated modified TALP medium containing 3 $\mathrm{mg} / \mathrm{ml} \mathrm{BSA}$ prior to ICSI.

Japanese monkey semen were collected from a sexually mature fertile male by electroejaculation. Samples were liquefied by incubation in a $\mathrm{CO}_{2}$ incubator at $37^{\circ} \mathrm{C}$ for $30 \mathrm{~min}$ and frozen by routine methods [16]. They were preserved in liquid nitrogen. Frozen semen were thawed just before use and an aliquot was washed in BWW with $3 \mathrm{mg} / \mathrm{ml} \mathrm{BSA} \mathrm{(BWW/}$ $B S A$ ) and then incubated in BWW/BSA with $1 \mathrm{mM}$ Caffeine and $1 \mathrm{mM}$ dbcAMP for 90 min for capacitation [15].

Intracytoplasmic sperm injection of oocytes Holding pipettes were flame-polished and adjusted to 

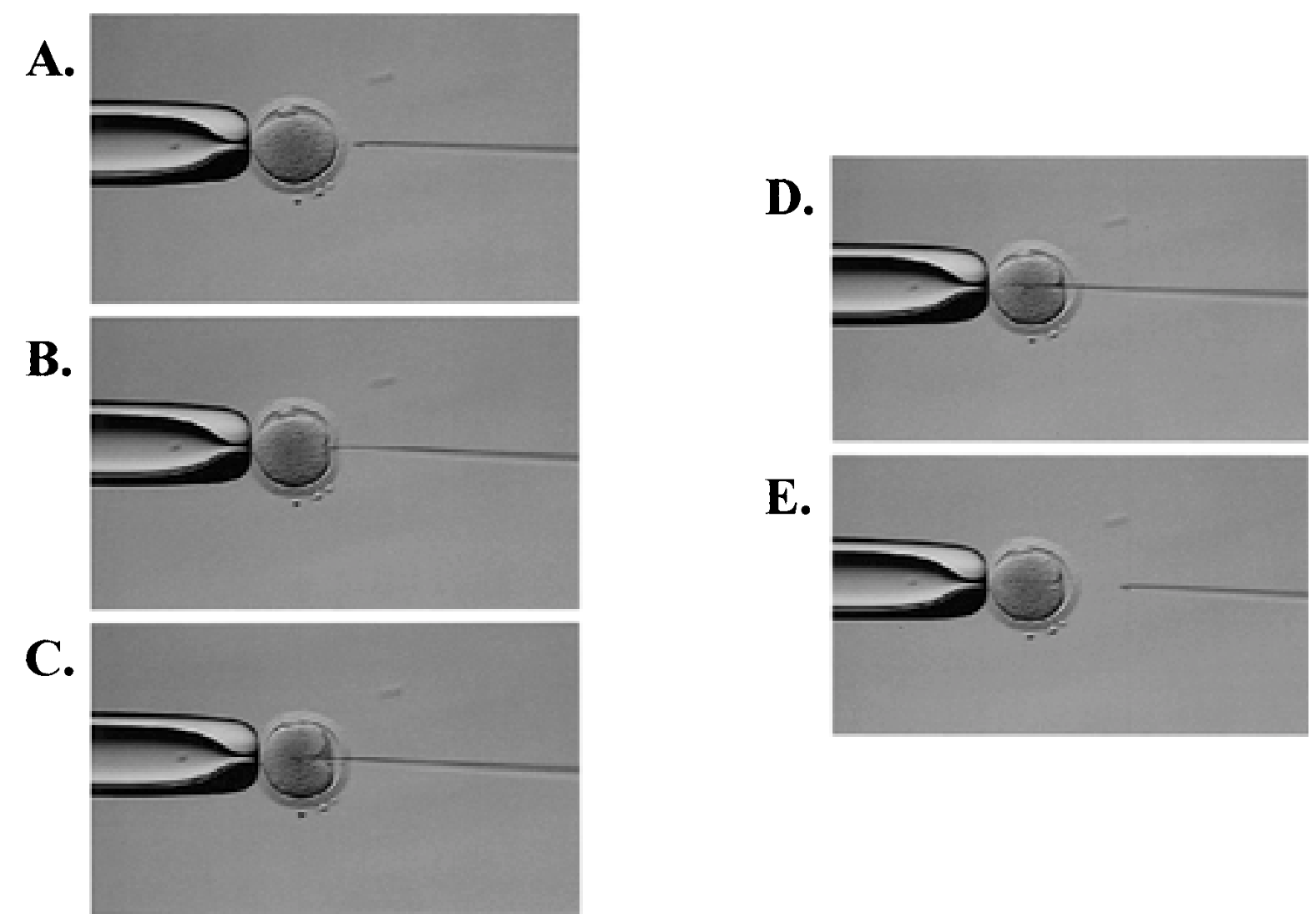

Fig. 2. Intracytoplasmic sperm injection (ICSI) procedure for the Japanese monkey. At first, motile spermatozoa were picked up in m-TALP medium and released in PVP medium. At the spot, membrane of sperm tail was broken by the rolling. A single spermatozoon aspirated from its tail end by the injection pipette in PVP spot.

(A) Oocyte was sucked by holding pipette in another spot. At that time, the polar body should be held at the 12 o'clock position. Injection procedure is important for subsequent developmental competence. (B, C) The injection pipette was introduced through the zona and pushed deeply into the oocyte cytoplasm causing a slight invagination of the oolemma. (D) The injection pipette was withdrawn to the center of the oocyte and then the cytoplasm was gently aspirated to ensure that the oolemma had been breached, at which point the oolemma resumed its shape. (E) The spermatozoon and aspirated cytoplasm were gently released into the center of the oocyte prior to removal of the injection pipette.

an external diameter of $100 \mu \mathrm{m}$ and internal diameter of $20 \mu \mathrm{m}$. Injection pipettes having an outer diameter of 6-7 $\mu \mathrm{m}$ and an internal diameter $4-5 \mu \mathrm{m}$ were bevelled to $30^{\circ}$. All manipulation procedures were performed at room temperature in $100 \mu \mathrm{l}$ TALP-HEPES under mineral oil (Sigma Chemical Co., Ltd., St Louis, MO, USA). Washed spermatozoa were diluted $1: 50$ in 10\% polyvinylpyrrolidone in saline (PVP: Sigma) to reduce motility. A single spermatozoon was aspirated from its tail end. Oocytes immobilized with the polar body in the 12 o'clock position were injected as previously described, as shown in Fig. 2 [17]. The injection pipette, containing a single spermatozoon, was introduced through the zona pellucida and pushed deeply into the oocyte cytoplasm causing a slight invagination of the oolemma. The injection pipette was withdrawn to the center of the oocyte and then the cytoplasm was gently aspirated to ensure that the oolemma had been breached, at which point the oolemma resumed its shape. The spermatozoon and aspirated cytoplasm were gently released into the center of the oocyte prior to removal of the injection pipette. The site of injection was barely visible within 1 min post-injection.

Assessment of fertilization, embryo transfer and embryo culture

Oocytes were examined between $18-20$ h post-sperm 
Table 1. Maturity of oocytes at time of collection

\begin{tabular}{cccccc}
\hline \multirow{2}{*}{ No. of Exp. } & $\begin{array}{c}\text { No. of oocytes } \\
\text { recovered }\end{array}$ & \multicolumn{4}{c}{ No. (\%) of oocytes } \\
\cline { 2 - 5 } & 125 & Deg. & GV & GVBD-MI & MII \\
\hline 7 & $4(3.2)$ & $54(43.2)$ & $23(18.4)$ & $44(35.2)$ \\
\hline
\end{tabular}

*Deg: degenerative oocytes, GV; germinal vesicle stage, MI: metaphase I, MII: metaphase II. **All oocytes were observed under a phase contrast microscope $(\times 100)$.

Table 2. Maturity of immature oocytes at $24 \mathrm{~h}$ after in vitro maturation

\begin{tabular}{lccccc}
\hline \multirow{2}{*}{$\begin{array}{c}\text { Maturity } \\
\text { at recovery }\end{array}$} & $\begin{array}{c}\text { No. of oocytes } \\
\text { Cultured }\end{array}$ & \multicolumn{4}{c}{ No. (\%) of oocyts } \\
\cline { 3 - 6 } & Gegenerate & GV & GVBD-MI & MII \\
\hline GV & 54 & $12(22.2)$ & $35(64.8)$ & $4(7.4)$ & $1(1.9)$ \\
GVBD-MI & 23 & $2(8.7)$ & - & $13(56.5)$ & $8(34.8)$ \\
\hline
\end{tabular}

*Deg: degenerative oocytes, GV; germinal vesicle stage, MI: metaphase I, MII: metaphase II. ** All oocytes were observed under a phase contrast microscope $(\times 100)$.

injection using Hoffman modulation contrast optics and the number of pronuclei stage oocytes was recorded. Oocytes containing two pronuclei and two polar bodies at $18-20 \mathrm{~h}$ after ICSI were considered to be fertilized. They were maintained in culture until the 2-cell stage (24-28 h post-injection). Some embryos were transferred to the oviducts of synchronous recipients under laparoscopic observation. The other embryos were cultured in CMRL with $10 \%$ heat inactivated fetal bovine serum (FBS: Invitrogen Corp., Carlsbad,USA) at $39^{\circ} \mathrm{C}$ in $5 \% \mathrm{CO}_{2}, 5 \% \mathrm{O}_{2}, 90 \% \mathrm{~N}_{2}$ and scored for development 7 days after sperm injection.

\section{Results}

A total of 125 oocytes were retrieved by laparoscopy from eight females at $40 \mathrm{~h}$ after hCG injection. Oocyte maturity at the time of collection was as follows (Table 1). Fifty-four (43.2\%) oocytes had an intact germinal vesicle (GV). Twenty-three (18.4\%) oocytes had undergone germinal vesicle breakdown (GVBD). Fourty-four (35.2\%) oocytes had reached metaphase II and 4 of them $(9.0 \%)$ were degenerated. Immmature oocytes were cultured for $24 \mathrm{hrs}$. Only $1.9 \%$ of GV oocytes (1/54) and $34.8 \%$ of GVBD-MI oocytes (8/23) reached the MII stage (Table 2 ).

Intracytoplasmic sperm injection was performed with metaphase II oocytes within $6 \mathrm{~h}$ post-isolation from follicles. Out of 40 metaphase-Il oocytes, 39 (97.5\%) survived after the injection and culture for $15 \mathrm{~h}$. Thirtyone of them $(79.5 \%)$ had an extruded second polar body (PB) and developed two distinct pronuclei (Fig. 3). Within 18-20 h post-ICSI (day 1), zygotes had completed the first mitosis resulting in two, sometimes unequal, daughter blastomeres (Fig. 3), often associated with small anucleate fragments. Nine MII oocytes derived from an immature condition at recovery were fertilized by ICSI and cultured. Eight oocytes with pronuclei were confirmed, however none of them cleaved.

Following the first mitosis, 7 cleaved stage embryos were transferred into the oviducts of four recipients. However, no pregnancies were established. The other embryos were transferred to CMRL medium and cultured to assess their in vitro developmental competence. After $168 \mathrm{~h}$ of ICSI, 6 morphologically normal blastocysts were observed. The rest of the ICSI embryos reached mostly the 16-32 cell stage and 6 morulae (data not shown). Blastocysts continued to expand for several days and hatched from zone pellucida (Fig. 3).

\section{Discussion}

The present results demonstrated that there are several features of Japanese macaque intracytoplasmic sperm injection that were improved using in vivo matured oocytes collected from gonadotrophinstimulated ovaries when compared to ICSI of in vitro matured oocytes in additional culture. As shown here, in vitro matured oocytes were less developmentally competent. The difference in developmental rates after ICSI might be due to quality of oocytes. Oocytes of Rhesus macaques matured in vitro from the germinal vesicle stage often display cytoskeletal abnormalities following fertilization by both IVF and ICSI, such as the 

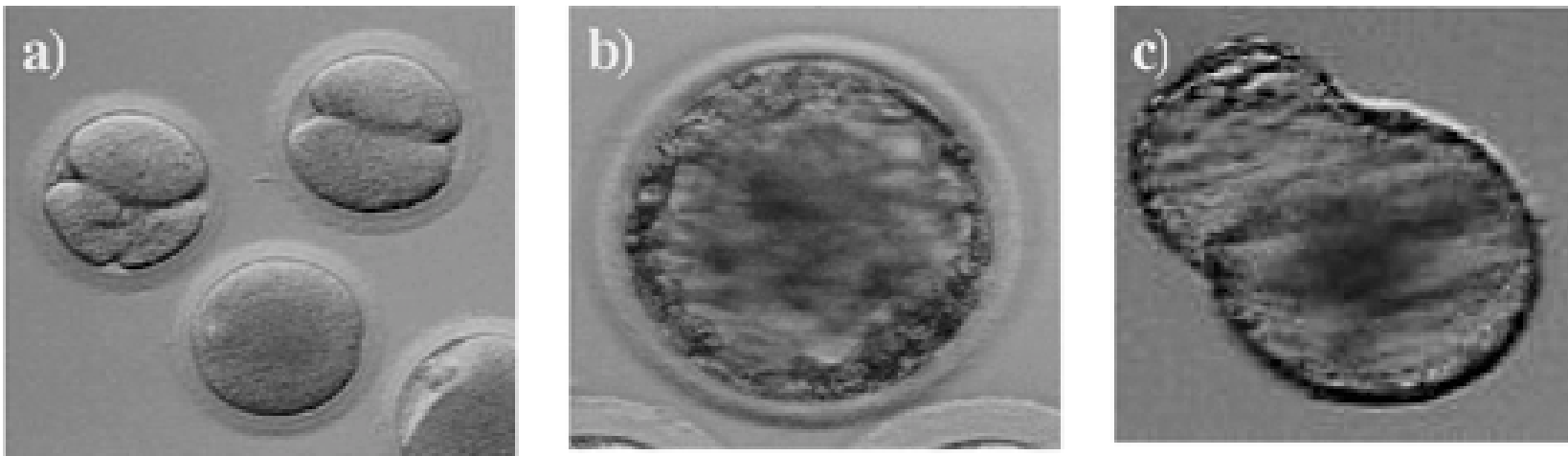

Fig. 3. Development of embryos derived from ICSI. ICSI embryos developed to pronuclear stage, 2cell, 3 cell stage at 18 - 20 hours after ICSI (a) and blastocyst at168 hours after culture in vitro (b). Hatched blastocysts were observed over 168 hours after culture in vitro (c).

Table 3. Results of ICSI in Japanese monkey

\begin{tabular}{lr}
\hline No. of experiments & 7 \\
No. of animals used & 8 \\
No. of cycles stimulated & 9 \\
No. of oocytes recovered & 125 \\
\hline No. of oocytes injected & 40 \\
No. of oocytes with PN (\%) & $31 \quad(78)$ \\
No. of embryos cultured (for 168 h after ICSI) & 22 \\
No. of embryos developing to blastocysts in vitro (\%) & $6 \quad(27)$ \\
\hline No. of embryos transferred (at 24 h after ICSI) & $9 *$ \\
No. of recipients & \multicolumn{2}{c}{4} \\
Pregnancy established & none \\
\hline
\end{tabular}

*Two embryos were lost in transfer.

inability to exit metaphase of meiosis, meiotic arrest following the resumption of meiosis and the formation of multiple female pronuclei after oocyte activation [14, 18]. Thus, the maturational failure observed after oocytes collected from stimulated females in this study might have been due to low quality oocytes. It might have been caused by an overdose of PMSG administration which can cause hormone imbalance in follicles or early removal of cumulus cells from immature oocytes.

In the Japanese monkey, additional stimulation after ICSI was not necessary for further development when in vivo matured oocytes were used (Table 3). Japanese monkey spermatozoa used for ICSI were obtained from fertile males and displayed normal semen characteristics in terms of morphology and motility. In an ICSI procedure, the additional rolling of the spermatozoa was necessary to achieve fertilization of Japanese macaque. In rhesus monkeys, ICSI of mature oocytes obtained from stimulated females did not need other additional chemical stimuli for oocyte activation other than microinjection of a spermatozoon [3]. The technical difference of ICSI between rhesus and Japanese macaques is the 'rolling' of the spermatozoa to immobilize them prior to injection. This procedure may result in modifications of the sperm plasma membrane facilitating subsequent sperm decondensation in the cytoplasm after ICSI [19]. Performing ICSI using spermatozoa with poor semen characteristics will make it possible to address whether rolling of spermatozoa prior to ICSI improves the rate of fertilization when using rhesus macaque compromised spermatozoa.

In the present study, ICSI of mature oocytes obtained from stimulated females resulted in activation without additional chemicals and development in CMRL with $10 \%$ FCS medium. However ICSI of oocytes matured in vitro resulted in activation of oocytes without additional 
chemicals, but not subsequent development. In contrast, it was reported that incubation in calcium ionophore was necessary to induce activation of in vitro matured rhesus oocytes collected from unstimulated females after performing ICSI [14]. Payne et al. [20] found that good quality embryos arose from oocytes that had more uniform timing from injection to pronuclear abuttal and tended to have a longer cytoplasmic wave. In the case of in vitro matured oocytes, the oocytes could not keep their activated status for fertilization and might need additional stimuli for a longer cytoplasmic wave of normal activation and further development in vitro.

Microtuble-mediated motility is particularly important for oocytes to be successfully fertilized by ICSI, since the spermatozoon deposited in a central position migrates to the oocyte cortex prior to first mitosis in the rhesus monkey [3, 14]. In oocytes fertilized by IVF, the incorporated spermatozoon remains at the oocyte cortex during pronuclear formation and apposition of male and female pronuclei $[13,18]$. Although the morphological difference of early fertilization between IVF and ICSI has been reported, the timing of fertilization and subsequent embryo development after ICSI was similar to that of oocytes fertilized by IVF and embryos cultured under the same conditions used in this study [16]. Early cleavage of rhesus IVF embryos in complex media without co-culture were successful, but further development of embryos was arrested at the morula stage or only a reduced rate of blastocyst formation was obtained [21]. In the case of rhesus embryos co-cultured with buffalo rat liver (BRL) monolayers, these cells supported development to the blastocyst stage at a higher rate. Rhresus monkey embryos obtained by ICSI were co-cultured in a complex medium on a BRL monolayer, and then underwent successive cleavage divisions resulting in large and hatched blastocysts 10 days post-ICSI. In our experiment, we observed that $27.3 \%$ of fertilized oocytes reached the blastocyst stage and some embryos arrested at the morula stage in our culture system. Furthermore ICSI embryos of Japanese monkeys developing to hatched blastocysts without any supporting cells (Fig. 3). The reason for the higher development rate of Japanese monkey embryos developing to the blastocyst stage in this study compared to the results of experiments on rhesus monkeys [3] is unclear. Possibly it is due to the procedure of ICSI discussed above.

Recently, rhesus IVF embryos were cultured to the blastocyst stage in a chemically defined and protein- free culture medium, and their developmental potential improved if the embryos were cultured in a complex medium from the 8- to 12-cell stage onwards [22]. As the same study reported that culture medium affected in vitro development of embryos, the developmental arrest observed in the study might be caused by sub-optimal conditions of culture in vitro.

In conclusion, we showed that oocytes retrieved by laparoscopy from gonadotrophin-stimulated Japanese monkeys resulted in high rates of fertilization after intracytoplasmic sperm injection (ICSI) and did not need additional chemical stimulus to induce oocyte activation. Subsequent culture of Japanese monkey ICSI embryos in CMRL with $10 \%$ FCS medium culminated in hatched blastocysts 9 days after ICSI. However the ability of Japanese monkey embryos fertilized by ICSI to be implanted, sustain a pregnancy and give rise to offspring were not demonstrated in this experiment. Success in Japanese monkey embryo propagation by ICSI will permit evaluation of the safety and biomedical basis of assisted reproductive technologies, leading to the design of further improvements as well as exploration of the realities of current clinical, and other unconventional reproductive methods and claims for alternative reproductive strategies.

\section{Acknowledgements}

We are indebted to Mr. Takahiro Nakagawa for surgical expertise, to Dr. Makoto Takenoshita, Dr. Youji Takenoshita, Mr. Namiki Uemasu for monkey semen collection. The authors would also like to acknowledge the helpful comments of Dr. Takashi Nagai. All animal procedures were approved by Research Institute of Experimental Animal, Shiga University of Medical Science, Institutional Animal Care and Use Regulations. This research was supported by Grant-In-Aid for Scientific Research from Japanese Ministry of Education, Culture, Sports Science and Technology.

\section{References}

1) Palermo, G., Joris, H., Devroey, P. and Van Steirteghem A.C. (1992): Pregnancies after intracytoplasmic sperm injection of single spermatozoon into an oocyte. Lancet, 340, 17-18.

2) Hosoi, Y., Miyake, M., Utsumi, K. and Iritani A. (1988): Development of rabbit oocytes after microinjection of spermatozoon. Proc. 11th Congr. Anim. Reprod. Artif. Insem. 3, 331-333.

3) Hewitson, L., Takahashi,D., Dominko, T., Simerly, C. and Schatten G. (1998) Fertilization and embryo development 
to blastocysts after intracytplasmic sperm injection in the rhesus monkey. Hum Reprod. 13, 3449-3455.

4) Gomez, M.C., Catt, J., Evans,G. and Maxwell, W.M. (1998): Cleavage, development and competence of sheep embryos fertilized by intracytoplasmic sperm injection and in vitro fertilization. Theriogenology, 49, 1143-1154.

5) Wu, J., Carrel, D.T. and Wilcox, A.L. (2001): Development of in vitro-atured oocytes from porcine preantral follicles following intracytplasmic sperm injection. Biol. Reprod., $65,1579-1585$.

6) Goto, K., Kinoshita, A., Takuma, T.Y. and Ogawa, K. (1990): Fertilization by sperm injection in cattle. Theriogenology, 33, 238.

7) Grondahl, G., Hansen, T.H., Hossaini, A., Heinze, I., Greve, T. and Hyttel, P. (1997): Intracytoplasmic sperm injection of in-vitro matured equine oocytes. Biol. Reprod. 57, 1495-1501.

8) Kimura, Y. and Yanagimachi, R. (1995): Intracytoplasmic sperm injection in the mouse. Biol. Reprod., 52, 709-720.

9) Enders, A.C., Boatman. D., Morgan, P. and Bavister, B.D. (1989): Differentiation of blastocysts derived from in vitro fertilized rhesus monkey ova. Biol. Reprod., 41, 715-727.

10) Bavister, B.D., Boatman, D.E., Coliins, K. Dierschke, D.J. and Eisele, S.G. (1984): Birth of rhesus monkey infant after in vitro fertilization and nonsurgical embryo transfer. Proc. Natl. Acad. Sci. USA, 81, 2212-2222.

11) Boatman, D.E. (1987): In vitro growth of non-human primate pre- and peri-implantation embryos. In: The Mammalian Preimplantation Embryo (Bavister, B.D., ed.) pp. 273-308, Plenum Press, New York.

12) Lanzendorf, S.E., Zelinski-Wooten, M.B., Stouffer, R.L. and Wolf D.P. (1990): Maturity at collection and the developmental potential of rhesus monkey oocytes. Biol. Reprod., 42, 703-711.

13) Wu, G.J., Simerly, C., Zoran, S.S., Funte, L.R. and Schatten, G. (1998): Microtubule and chromatin dynamics during fertilization and early development in rhesus monkeys, and regulation by intracellular calcium ions. Biol. Reprod., 55, 260-270.

14) Hewitson, L., Simerly, C., Tengowski, M.W., Sutovsky, P., Navara, C.S., Haavisto, A.J. and Schatten, G. (1996):
Microtubule and chromatin configurations during rhesus intracytoplasmic sperm injection: successes and failures. Biol. Reprod., 55, 271-280.

15) Torii, R., Hosoi, Y., Masuda, Y., Iritani, A. and Nigi, H. (2000): Birth of the Japanese monkey (Macaca fuscata) infant following in-vitro fertilization and embryo transfer. Primates, 41, 39-47.

16) Sankai, T., Terao, K., Yanagunachi, R., Cho, F. and Yoshikawa, Y. (1994): Cryopreservation of spermatozoa from cynomolgus monkey (Macaca fascicularis). J. Reprod. Fertil., 101, 273-278.

17) Hosoi, Y., Torii, R. and Iritani, A. (1998): An experimental model of in vitro fertilization by microinjection of spermatozoa for preservation of endangered species. In: Reproductive Biology Update (Miyamoto, H. and Manabe, N. eds.) pp. 377-381, Nakanishi Printing Co., Ltd., Kyoto, Japan.

18) Sutovsky, P., Hewitson, L., Simerly, C. and Schatten, G. (1996): Intracytoplasmic sperm injection for rhesus monkey fertilization results in unusual chromatin, cytoskeletal and membrane events, but eventually leads to pronuclear development and sperm aster assembly. Hum. Reprod., 11, 1703-1712.

19) Fishel, S., Lisi, F., Rinaldi, L., Green S., Hunter A., Dowell, K. and Thornton, S. (1995): Systematic examination of immobilizing spermatozoa before intracytoplasmic sperm injection in the human. Hum. Reprod., 10, 407-500.

20) Payne, D., Flaherty, S.P., Barry, M.F., Matthews, C.D. (1997): Preliminary observations on polar body extrusion and pronuclear formation in human oocytes using timelapse video cinematography. Hum Reprod., 12, 532-541.

21) Bavister, B.D., Boatman, D.E., Leibfried, L., Loose, M. and Vernon, M.W. (1983): Fertilization and cleavage of rhesus monkey oocytes in vitro. Biol. Reprod., 28, 983999.

22) Schramm, R.D. and Bavister, B.D. (1996): Development of in-vitro fertilized primate embryos into blastocysts in a chemically defined, protein-free culture medium. Hum. Reprod., 11, 1690-1697. 